\title{
INTEGRAL FORMULAS WITH WEIGHT FACTORS
}

\author{
TELEMACHOS HATZIAFRATIS
}

(Received 5 June 1989; revised 25 October 1989)

Communicated by Iain Raeburn

\begin{abstract}
A Bochner-Martinelli-Koppelman type integral formula with weight factors is derived on complete intersection submanifolds of domains of $\mathbb{C}^{n}$.

1991 Mathematics subject classification (Amer. Math. Soc.) 32 A 25.
\end{abstract}

\section{Introduction}

A large part of complex analysis deals with problems of constructing holomorphic functions and more general analytic objects (differential forms, sections of holomorphic vector bundles etc.) One of the most important tools for such constructions is the $\bar{\partial}$-equation (see for example Hörmander [11] and Henkin and Leiterer [9]). The version of the $\bar{\partial}$-equation that one has to study and the techniques to solve it, depend on the particular problem.

In recent years various forms of the $\bar{\partial}$-equation (mostly on the complexanalytic side of the subject) have been successfully studied using integral formulas. These formulas are several complex variable analogues of Cauchy's integral formula:

If $D \subset \mathbb{C}$ is a bounded domain in the complex plane with smooth boundary $\partial D$ then for $f \in C^{1}(\bar{D})$ and $z \in D$ we have

$$
f(z)=\frac{1}{2 \pi i}\left[\int_{\partial D} f(\zeta) \frac{d \zeta}{\zeta-z}-\iint_{D} \bar{\partial} f(\zeta) \wedge \frac{d \zeta}{\zeta-z}\right]
$$

(see [11, p.3]); this is known as Ponpeju representation formula.

(C) 1992 Australian Mathematical Society 0263-6115/92\$A2.00+0.00 
It follows from (1) that for a bounded function $f \in C^{1}(D)$ we have

$$
f(z)=\frac{\partial}{\partial \bar{z}}\left[\frac{1}{2 \pi i} \iint_{D} f(\zeta) \frac{d \zeta \wedge d \bar{\zeta}}{\zeta-z}\right], z \in D
$$

(see $[11$, p. 3$])$; in particular (2) gives a solution $g$ of the equation $\partial g / \partial \bar{z}=$ $f$.

Generalizations of (1) and (2) to several variables consist in constructing kernels $K_{q}$ so that

$$
f=\int_{\partial D} f \wedge K_{q}-\int_{D} \bar{\partial} f \wedge K_{q}+\bar{\partial}\left(\int_{D} f \wedge K_{q-1}\right)
$$

for appropriate domains $D \subset \mathbb{C}^{n}$ and $(0, q)$-forms $f$ in $D$ (see, for example, Ovrelid [12]). These are known as Bochner-Martinelli-Koppelman type formulas and they can be modified to produce a variety of other formulas with which the $\bar{\partial}$-equation can be studied (see, for example, Henkin and Leiterer [9, 10] and Range [13]).

More relevant to this paper is the work of Andersson and Berndtsson [2] who constructed integral kernels with weight factors producing, in this way, a large class of integral formulas. An example of (2) with weights is

$$
f(z)=\frac{\partial}{\partial \bar{z}}\left[\frac{1}{2 \pi i} \iint_{D} F(\zeta, z) f(\zeta) \frac{d \zeta \wedge d \bar{\zeta}}{\zeta-z}\right]
$$

where $F(\zeta, z)$ in any $C^{1}$-function which is holomorphic in $z$ and $F=1$ if $\zeta=z$ (see [2, p. 93]). If $F \equiv 1$ then (3) becomes (2).

In this paper we generalize the construction of Andersson and Berndtsson from domains of $\mathbb{C}^{n}$ to submanifolds of domains $\mathbb{C}^{n}$ which arise as complete intersections. There are several applications of weighted integral formulas; see for example Berndtsson [3], Charpentier [5], Dantov and Henkin [6] and Skoda [14]. Berndtsson [4] recently used the construction of [2] to obtain integral formulas in domains of complex projective space and then applied these to study the Radon-Penrose transform. In fact we developed our integral formulas in the setting of this paper, trying to generalize the work of [4] to the case of algebraic manifolds (complete intersections in domains of complex projective space).

NotATION. We will use determinants with entries differential forms: if $a_{i j}$ are differential forms then

$$
\operatorname{det}\left[a_{1 j}, \ldots, a_{n j}\right]=\sum_{\sigma} \operatorname{sign}(\sigma) a_{1 \sigma(1)} \wedge \ldots \wedge a_{n \sigma(n)}
$$

where the summation is extended over all permutations $\sigma$ of $\{1, \ldots, n\}$. Thus when we write $\operatorname{det}\left[a_{1 j}, \ldots, a_{n j}\right]$ we mean that $j$ runs from $j=1$ to 
$j=n$ forming the $n$-rows of the determinant. We will also write the same determinant as

$$
\operatorname{det}\left[\begin{array}{l}
a_{11} \ldots a_{n 1} \\
a_{1 j} \ldots a_{n j}
\end{array}\right]
$$

here we mean that $j$ runs from $j=2$ to $j=n$ forming the 2 nd up to the $n$th row. If we put an integer above a column we mean that this column is to be repeated in the determinant as many times as the integer indicates. For properties of such determinants see $[1$, p. 8$]$.

\section{Statement of the results}

Let $D, \Omega \subset \mathbb{C}^{n}$ be bounded domains with smooth boundary so that $\bar{D} \subset \Omega$. Let $h: \Omega \rightarrow \mathbb{C}^{p}(p<n)$ be a holomorphic map and set $V=\{\zeta \in \Omega$ : $h(\zeta)=0\}, M=V \cap D$ and $\partial M=V \cap(\partial D)$. Assume that $d h_{1} \wedge \ldots \wedge d h_{p} \neq 0$ on $\bar{M}$ and that $V$ meets $\partial D$ transversally so that $M$ is a complex manifold of (complex) dimension $n-p$ and $\partial M$ is a smooth manifold of (real) dimension $2 n-2 p-1$. Let $h_{l j} \in \mathscr{O}(\Omega \times \Omega)(1 \leq l \leq p, 1 \leq j \leq n)$ be holomorphic functions in $(\zeta, z) \in \Omega \times \Omega$ so that

$$
h_{l}(\zeta)-h_{l}(z)=\sum_{j=1}^{n} h_{l j}(\zeta, z)\left(\zeta_{j}-z_{j}\right)
$$

Consider a $C^{1}$-map $s: \bar{D} \times \bar{D} \rightarrow \mathbb{C}^{n}$ so that

$$
\langle s, \zeta-z\rangle=\sum_{j=1}^{n} s_{j}(\zeta, z)\left(\zeta_{j}-z_{j}\right) \neq 0 \quad \text { for } \zeta \neq z .
$$

Moreover assume that

$$
|s(\zeta, z)|=O(|\zeta-z|) \text { and }\left|\langle s, \zeta-z\rangle^{-1}\right|=O\left(|\zeta-z|^{-2}\right)
$$

uniformly in $\zeta \in \bar{D}$ and $z$ in compact subsets of $D$. Also let $Q: \bar{D} \times \bar{D} \rightarrow$ $\mathbb{C}^{n}$ be a $C^{1}$-map and $G(w)$ a holomorphic function of $w$ defined in a neighborhood of the image of the function $\bar{D} \times \bar{D} \ni(\zeta, z) \mapsto\langle Q(\zeta, z), \zeta-$ $z\rangle \in \mathbb{C}$, with $G(0)=1$ (see below for examples). In this setting we introduce the following kernels:

$$
\begin{aligned}
\widetilde{K}= & c \sum_{k=0}^{n-p-1} \frac{(-1)^{k}}{k !} G^{(k)}(\langle Q, \zeta-z\rangle)(\langle s, \zeta-z\rangle)^{-(n-p-k)} \\
& \times \operatorname{det}[h_{1 j}, \ldots, h_{p j}, s_{j}, \overbrace{\bar{\partial} Q_{j}}^{k}, \overbrace{\bar{\partial} s_{j}}^{n-p-k-1}]
\end{aligned}
$$


(defined for $\zeta \neq z$ ) and

$$
\widetilde{P}=c \frac{(-1)^{n-p}}{(n-p) !} G^{(n-p)}(\langle Q, \zeta-z\rangle) \operatorname{det}[h_{1 j}, \ldots, h_{p j}, \overbrace{\bar{\partial} Q_{j}}^{n-p}]
$$

where

$$
c=(-1)^{\frac{(n-p)(n-p-1)}{2}} 1 /\left((2 \pi i)^{n-p}\right) \cdot 1 /((n-p) ! ;
$$

here $\bar{\partial}=\bar{\partial}_{\zeta}+\bar{\partial}_{z}$. Also define $\beta=\beta(\zeta)$ as follows:

$$
\beta(\zeta)=|\nabla h|^{-2} \operatorname{det}[\frac{\overline{\partial h_{1}}}{\partial \zeta_{j}}, \ldots, \frac{\overline{\partial h_{p}}}{\partial \zeta_{j}}, \overbrace{d \zeta_{j}}^{n-p}]
$$

where

$$
|\nabla h|=\left(\sum_{1 \leq j_{1}<\ldots<j_{p} \leq n}\left|\frac{\partial\left(h_{1}, \ldots, h_{p}\right)}{\partial\left(\zeta_{j 1}, \ldots, \zeta_{j_{p}}\right)}\right|^{2}\right)^{1 / 2} .
$$

With $K(\zeta, z)=\widetilde{K}(\zeta, z) \wedge \beta(\zeta)$ and $P(\zeta, z)=\widetilde{P}(\zeta, z) \wedge \beta(\zeta)$ let $K_{q}(\zeta, z)$ be the part of $K(\zeta, z)$ which is a $(0, q)$-form in $z$ and $(n-p, n-p-q-1)$ form in $\zeta$. Similarly define $P_{q}(\zeta, z)$ from $P(\zeta, z)$. More precisely

$$
K_{q}=c \sum_{k=0}^{n-p-1} \sum_{m=0}^{q} \frac{(-1)^{k}}{k !} G^{(k)}(\langle Q, \zeta-z\rangle)^{-(n-p-k)} T_{k, m, q}(\zeta, z) \wedge \beta(\zeta)
$$

where

$$
\begin{aligned}
T_{k, m, q}= & \left(\begin{array}{c}
k \\
m
\end{array}\right)\left(\begin{array}{c}
n-p-k-1 \\
q-m
\end{array}\right) \\
& \times \operatorname{det}[\overbrace{h_{l j}}^{1 \leq l \leq p}, s_{j}, \overbrace{\partial_{z} Q_{j}}^{m}, \overbrace{\partial_{\zeta} Q_{j}}^{k-m}, \overbrace{\bar{\partial}_{z} s_{j}}^{q-m}, \overbrace{\overbrace{\partial_{z} s_{j}}^{n-p-q-k+m-1}}^{n-m}]
\end{aligned}
$$

and

$$
P_{q}=c \frac{(-1)^{n-p}}{(n-p) !} G^{(n-p)}(\langle Q, \zeta-z\rangle)\left(\begin{array}{c}
n-p \\
q
\end{array}\right) \operatorname{det}[\overbrace{h_{l j}}^{1 \leq l \leq p}, \overbrace{\bar{\partial}_{z} Q_{j}}^{q}, \overbrace{\bar{\partial}_{\zeta} Q_{j}}^{n-p-q}] \wedge \beta(\zeta)
$$

Also let $K_{-1} \equiv 0$.

The main result of this paper is the following Bochner-Martinelli-Koppelman type formula. 
THEOREM 1. Let $f \in\left(C^{1}(\bar{M})\right)_{(0, q)}$, that is $(0, q)$-form with coefficients in $C^{1}(\bar{M})$. Then for a $z \in M$,

$$
\begin{aligned}
f(z)= & \int_{\zeta \in \partial M} f(\zeta) \wedge K_{q}(\zeta, z)-\int_{\zeta \in M} \bar{\partial} f(\zeta) \wedge K_{q}(\zeta, z) \\
& +\bar{\partial}_{z}\left(\int_{\zeta \in M} f(\zeta) \wedge K_{q-1}(\zeta, z)\right)-(-1)^{q} \int_{\zeta \in M} f(\zeta) \wedge P_{q}(\zeta, z) .
\end{aligned}
$$

Observing that $P_{q}=0$ for $q \geq 1$ if $Q(\zeta, z)$ is holomorphic in $z$, we obtain from Theorem 1 the following.

Theorem 2. If $Q(\zeta, z)$ is holomorphic in $z$ then for

$$
f \in\left(C^{1}(\bar{M})\right)_{(0, q)} \quad(q \geq 1)
$$

we have

$$
f(z)=\int_{\partial M} f \wedge K_{q}-\int_{M} \bar{\partial} f \wedge K_{q}+\bar{\partial}_{z}\left(\int_{M} f \wedge K_{q-1}\right)
$$

for $z \in M$.

THeOREM 3. Suppose that $Q(\zeta, z)$ is holomorphic in $z$ and that $s(\zeta, z)$ is also holomorphic in $z$ for each $\zeta \in \partial M$. Then for $f \in(C(\bar{M}))_{(0, q)}(q \geq 1)$ with $\bar{\partial} f=0$ we have, in the sense of distributions, the following equation

$$
\bar{\partial}_{z}\left(\int_{M} f \wedge K_{q-1}\right)=f \text {. }
$$

EXAMPLES. (1) A simple choice for $s$ is $s_{j}=\bar{\zeta}_{j}-\bar{z}_{j}$, the map $Q$ is quite general and as for $G$ one may choose, for example, any entire function of $w \in \mathbb{C}$ with $G(0)=1$; for example $G(w)=e^{w}$.

(2) Suppose $\varphi$ is negative convex function on $\Omega$ and set

$$
Q_{j}=-\frac{1}{\varphi(\zeta)} \frac{\partial \varphi}{\partial \zeta_{j}}(\zeta)
$$

Then $\operatorname{Re}(\langle Q, \zeta-z\rangle+1)>0$ (see [2, p. 101]). Hence one may choose $G(w)=(1+w)^{-N}, N>0$. (For more details on this example and other examples of choices of $s, G$ and $Q$ see [2, pp. 101-108]).

ReMARKs. (1) Let us point out that, in view of Sard's Theorem, the assumptions that $M$ be smooth and that $V$ meet $\partial D$ transversally are satisfied generically, in the sense that given $V$ and $D$, arbitrarily small perturbations of the system $(V, \partial D)$ will satisfy these assumptions.

(2) The case $Q \equiv 0$ (or $G \equiv 1$ ) and $s_{j}=\bar{\zeta}_{j}-\bar{z}_{j}$ for $|\zeta-z|<$ small constant, is in [8]. In fact the proof of Theorem 1 will be reduced to this case. 
(3) The case $p=0$, that is, when $M=D$ is in [2]; although the kernels there are introduced differently, they coincide with ours (for $p=0$ ).

\section{Preparation for the proof}

The proof of Theorem 1 will involve two steps: one is an application of Stokes's Theorem and the other is a process of passing to a residue. For the first part we will need the following lemmas.

LEMMA 1. If $\varphi$ is a smooth function then

$$
\operatorname{det}[\varphi s_{j}, \overbrace{\bar{\partial} s_{j}}^{l}, * * *]=\varphi^{l+1} \operatorname{det}[s_{j}, \overbrace{\bar{\partial} s_{j}}^{l}, * * *]
$$

where $* * *$ denote some appropriate terms which are the same on both sides of the equation.

Proof. Since

$$
\bar{\partial}\left(\varphi s_{j}\right)=\varphi \bar{\partial} s_{j}+s_{j} \bar{\partial} \varphi
$$

we obtain

$$
\begin{aligned}
& \operatorname{det}[\varphi s_{j}, \overbrace{\bar{\partial}\left(\varphi s_{j}\right)}^{l}, * * *]=\operatorname{det}[\varphi s_{j}, \overbrace{\varphi \bar{\partial} s_{j}+s_{j} \bar{\partial} \varphi}^{l}, * * *] \\
& =\operatorname{det}[\varphi s_{j}, \overbrace{\varphi \bar{\partial} s_{j}}^{l}, * * *]+\left(\text { terms of the form } \operatorname{det}\left[\varphi s_{j}, s_{j} \bar{\partial} \varphi, \ldots\right]\right)
\end{aligned}
$$

where the dots denote various terms.

Now the identity of the lemma follows from the above equation since

$$
\operatorname{det}[\varphi s_{j}, \overbrace{\varphi \bar{\partial} s_{j}}^{l}, * * *]=\varphi^{l+1} \operatorname{det}[s_{j}, \overbrace{\bar{\partial} s_{j}}^{l}, * * *]
$$

and

$$
\operatorname{det}\left[\varphi s_{j}, s_{j} \bar{\partial} \varphi, \ldots\right]= \pm \varphi \operatorname{det}\left[s_{j}, s_{j} \ldots\right] \wedge \bar{\partial} \varphi=0
$$

LEMMA 2. For differential forms restricted (in $(\zeta, z)$ ) to $M \times M-\{\zeta=z\}$ we have

$$
\bar{\partial} K=\left(\bar{\partial}_{\zeta}+\bar{\partial}_{z}\right) K=P .
$$

Proof. Let us consider the term

$$
A_{k}=G^{(k)}(\langle Q, \zeta-z\rangle)(\langle s, \zeta-z\rangle)^{-(n-p-k)} \operatorname{det}[h_{1 j}, \ldots, h_{p j}, s_{j}, \overbrace{\bar{\partial} Q_{j}}^{k}, \overbrace{\bar{\partial} s_{j}}^{n-p-k-1}]
$$


$(\zeta \neq z)$ and write it as

$$
A_{k}=G^{(k)}(\langle Q, \zeta-z\rangle) \operatorname{det}[h_{1 j}, \ldots, h_{p j}, \gamma_{j}, \overbrace{\bar{\partial} Q_{j}}^{k} \overbrace{\bar{\partial} \gamma_{j}}^{n-p-k-1}]
$$

where $\gamma_{j}=\langle s, \zeta-z\rangle^{-1} s_{j}$ so that

$$
\langle\gamma, \zeta-z\rangle=1
$$

The fact that we can write $A_{k}$ in this way follows form Lemma 1 , applied with $\varphi=\langle s, \zeta-z\rangle^{-1}$. In proving (1) we may assume $\zeta_{1} \neq z_{1}$; then

$$
\begin{aligned}
& \left(\zeta_{1}-z_{1}\right) A_{k}=G^{(k)}(\langle Q, \zeta-z\rangle)
\end{aligned}
$$

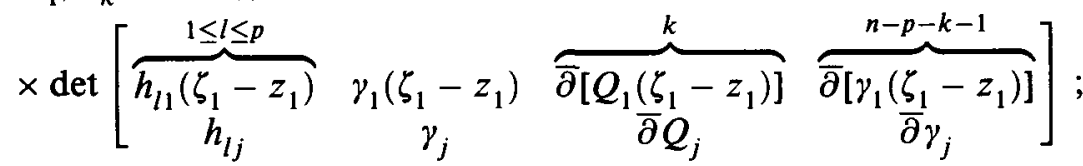

multiplying the $j$ th-rows of the above determinant by $\left(\zeta_{j}-z_{j}\right)(2 \leq j \leq n)$ and adding them to the first row we obtain, in view of (2) and the fact that $\zeta, z \in M$, that

$$
\left(\zeta_{1}-z_{1}\right) A_{k}=G^{(k)}(\langle Q, \zeta-z\rangle) \operatorname{det}\left[\begin{array}{cccc}
\overbrace{0}^{1 \leq l \leq p} & 1 & \overbrace{\bar{\partial}(\langle Q, \zeta-z\rangle)}^{k} & \overbrace{0}^{n-p-1} \\
h_{l j} & \gamma_{j} & \bar{\partial} Q_{j}
\end{array}\right] .
$$

Therefore

$$
\begin{aligned}
& \left(\zeta_{1}-z_{1}\right) \bar{\partial} A_{k}=G^{(k+1)}(\langle Q, \zeta-z\rangle) \bar{\partial}(\langle Q, \zeta-z\rangle) \\
& \wedge \operatorname{det}\left[\begin{array}{cccc}
\overbrace{0}^{1 \leq l \leq p} & 1 & \overbrace{\bar{\partial}(\langle Q, \zeta-z\rangle)}^{k} & \overbrace{0}^{n-p-k-1} \\
h_{l j} & \gamma_{j} & \bar{\partial} \gamma_{j}
\end{array}\right] \\
& +G^{(k)}(\langle Q, \zeta-z\rangle) \operatorname{det}\left[\begin{array}{cccl}
\overbrace{0}^{1 \leq l \leq p} & 0 & \overbrace{\bar{\partial}(\langle Q, \zeta-z\rangle)}^{k} & \overbrace{0}^{n-k-1} \\
h_{l j} & \bar{\partial} \gamma_{j} & \bar{\partial} Q_{j} & \bar{\partial} \gamma_{j}
\end{array}\right] .
\end{aligned}
$$


Expanding the determinants we obtain

$$
\begin{aligned}
\left(\zeta_{1}-z_{1}\right) \bar{\partial} A_{k}= & (-1)^{p} G^{(k+1)}(\langle Q, \zeta-z\rangle) \bar{\partial}(\langle Q, \zeta-z\rangle) \\
& \wedge \operatorname{det}[\overbrace{h_{l j}}^{1 \leq l \leq p}, \overbrace{\partial Q_{j}}^{k} \overbrace{\bar{\partial} \gamma_{j}}^{n-p-k-1}]_{2 \leq j \leq n} \\
& +(-1)^{p} k G^{(k)}(\langle Q, \zeta-z\rangle) \bar{\partial}(\langle Q, \zeta-z\rangle) \\
& \wedge \operatorname{det}[\overbrace{h_{l j}}^{1 \leq l \leq p}, \overbrace{\bar{\partial} Q_{j}}^{k-1}, \overbrace{\bar{\partial} \gamma_{j}}^{n-p-k}]_{2 \leq j \leq n}
\end{aligned}
$$

or equivalently,

$$
(-1)^{p}\left(\zeta_{1}-z_{1}\right) \bar{\partial}\left[\frac{(-1)^{k}}{k !} A_{k}\right]=B_{k+1}-B_{k} \quad(0 \leq k \leq n-p-1)
$$

where

$$
\begin{aligned}
& B_{k+1}= \frac{(-1)^{k}}{k !} G^{(k+1)}(\langle Q, \zeta-z\rangle) \bar{\partial}(\langle Q, \zeta-z\rangle) \\
& \wedge \operatorname{det}[\overbrace{h_{l j}}^{1 \leq l \leq p}, \overbrace{\bar{\partial} Q_{j}}^{k}, \overbrace{\bar{\partial} \gamma_{j}}^{n-p-k-1}]_{2 \leq j \leq n}
\end{aligned}
$$

for $0 \leq k \leq n-p-1$ and $B_{0}=0$.

Summing from $k=0$ to $k=n-p-1$ we obtain

$$
\begin{aligned}
\frac{1}{c}(-1)^{p}\left(\zeta_{1}-z_{1}\right) \bar{\partial} \widetilde{K}= & \sum_{k=0}^{n-p-1}\left(B_{k+1}-B_{k}\right)=B_{n-p} \\
= & \frac{(-1)^{n-p}}{(n-p-1) !} G^{(n-p)}(\langle Q, \zeta-z\rangle) \bar{\partial}(\langle Q, \zeta-z\rangle) \\
& \wedge \operatorname{det}[\overbrace{h_{l j}^{1 \leq p}}^{1 \leq \overbrace{}^{n-p-1} Q_{j}}]_{1 \leq j \leq n} .
\end{aligned}
$$


On the other hand

$$
\begin{aligned}
& \frac{1}{c}\left(\zeta_{1}-z_{1}\right) \widetilde{P}=\frac{(-1)^{n-p}}{(n-p) !} G^{(n-p)}(\langle Q, \zeta-z\rangle) \\
& \times \operatorname{det}[\overbrace{\begin{array}{c}
h_{l 1}\left(\zeta_{1}-z_{1}\right) \\
h_{l j}
\end{array}}^{1 \leq l \leq p} \overbrace{\frac{\partial}{\left.\partial Q_{1}\left(\zeta_{1}-z_{1}\right)\right]}}^{n-p}] \\
& =\frac{(-1)^{n-p}}{(n-p) !} G^{(n-p)}(\langle Q, \zeta-z\rangle) \operatorname{det}[\overbrace{0}^{1 \leq l \leq p}, \overbrace{\substack{\partial(\langle Q, \zeta-z\rangle) \\
h_{l j}}}^{n-p}] \\
& =(-1)^{p} \frac{(-1)^{n-p}}{(n-p-1) !} G^{(n-p)}(\langle Q, \zeta-z\rangle) \bar{\partial}(\langle Q, \zeta-z\rangle) \\
& \wedge \operatorname{det}[\overbrace{h_{l j}}^{1 \leq l \leq p} \overbrace{\bar{\partial} Q_{j}}^{n-p-1}]_{2 \leq j \leq n} .
\end{aligned}
$$

Now (3) and (4) prove that $\bar{\partial} \tilde{K}=\tilde{P}$. Moreover it follows from [7, Corollary 1] that $\bar{\partial} \beta(\zeta)=0$ and therefore $\bar{\partial}(\widetilde{K} \wedge \beta)=\widetilde{P} \wedge \beta$. This completes the proof of Lemma 2 .

\section{Proof of Theorem 1}

The integral formula of Theorem 1 will follow as soon as we prove that for $\varphi(z) \in\left(C_{0}^{1}(M)\right)_{(n-p, n-p-q)}$ we have

$$
\begin{aligned}
\int_{z \in M} f(z) \wedge \varphi(z)= & \int_{z \in M}\left(\int_{\zeta \in \partial M} f(\zeta) \wedge K_{q}(\zeta, z)\right) \wedge \varphi(z) \\
& -\int_{z \in M}\left(\int_{\zeta \in M}(\bar{\partial} f)(\zeta) \wedge K_{q}(\zeta, z)\right) \wedge \varphi(z) \\
& +\int_{z \in M}\left(\bar{\partial}_{z}\left(\int_{\zeta \in M} f(\zeta) \wedge K_{q-1}(\zeta, z)\right)\right) \wedge \varphi(z) \\
& -(-1)^{q} \int_{z \in M}\left(\int_{\zeta \in M} f(\zeta) \wedge P_{q}(\zeta, z)\right) \wedge \varphi(z) .
\end{aligned}
$$

By degree reasons (see the remark on page 44 of [9]) this is equivalent to

$$
\begin{aligned}
\int_{z \in M} f(z) \wedge \varphi(z) & =\int_{(\zeta, z) \in(\partial M) \times M} f \wedge K \wedge \varphi-\int_{m \times M} \bar{\partial} f \wedge K \wedge \varphi \\
& +\int_{M}\left(\bar{\partial}_{z}\left(\int_{M} f \wedge K\right)\right) \wedge \varphi-(-1)^{q} \int_{M}\left(\int_{M} f \wedge P\right) \wedge \varphi .
\end{aligned}
$$


Let us keep in mind the variables of integration and the way the various differential forms depend on them, that is

$$
\bar{\partial} f=\bar{\partial} f(\zeta), K=K(\zeta, z), P=P(\zeta, z), \varphi=\varphi(z) \text { and } f=f(\zeta)
$$

(unless otherwise indicated).

Since $\varphi$ has compact support in $M$ we have

$$
\int_{(\zeta, z) \in(\partial M) \times M} f \wedge K \wedge \varphi=\int_{\partial(M \times M)} f \wedge K \wedge \varphi .
$$

The singularities of $f \wedge K \wedge \varphi$ in (2) occur at $\zeta=z$ and the $(2 n-2 p-1)$ dimensional cycle $\partial(M \times M)$ surrounds " $\zeta=z$ "; and this will give the extra term (the residue term) in (1), namely $\int_{M} f \wedge \varphi$; the other terms in (1) arise from the "volume" integral in applying Stokes's theorem to the right-hand side of (2). Indeed, by Strokes's Theorem,

$$
\int_{\partial(M \times M)} f \wedge K \wedge \varphi=\int_{M \times M-\{|\zeta-z|<\varepsilon\}} d(f \wedge K \wedge \varphi)+\int_{C_{\varepsilon}} f \wedge K \wedge \varphi
$$

where $C_{\varepsilon}=\{(\zeta, z) \in M \times M:|\zeta-z|=\varepsilon\}$.

By Lemma 2 and degree reasons,

$$
\begin{aligned}
\lim _{\varepsilon \rightarrow 0} \int_{M \times M-\{|\zeta-z|<\varepsilon\}} d(f \wedge K \wedge \varphi)= & \int_{M \times M} \bar{\partial} f \wedge K \wedge \varphi+(-1)^{q} \int_{M \times M} f \wedge P \wedge \varphi \\
& +(-1)^{q+1} \int_{M \times M} f \wedge K \wedge \bar{\partial} \varphi .
\end{aligned}
$$

Since $\varphi=\varphi(z)$ has compact support in $M$, integration by parts gives,

$$
\int_{M \times M} f \wedge K \wedge \bar{\partial} \varphi=(-1)^{q} \int_{M}\left(\bar{\partial}_{z}\left(\int_{M} f \wedge K\right)\right) \wedge \varphi .
$$

Thus, in view of (2), (3), (4) and (5), the proof of Theorem 1 is reduced to showing that

$$
\lim _{\varepsilon \rightarrow 0} \int_{C_{\varepsilon}} f(\zeta) \wedge K(\zeta, z) \wedge \varphi(z)=\int_{z \in M} f(z) \wedge \varphi(z) .
$$

To prove $(I)$ we make two reductions: one to the case $Q \equiv 0$ and then another one to the case $Q \equiv 0$ and $s=b$ where $b_{j}=\bar{\zeta}_{j}-\bar{z}_{j}$.

ReDUCTION TO THE CASE $Q \equiv 0$. This will be made by proving the following

$$
\lim _{\varepsilon \rightarrow 0} \int_{C_{\varepsilon}} f(\zeta) \wedge \tilde{A}_{k}(\zeta, z) \wedge \varphi(z)=0 \text { for } 1 \leq k \leq n-p-1
$$

where $\widetilde{A}_{k}=A_{k} \wedge \beta\left(A_{k}\right.$ was defined in the proof of Lemma 1$)$ and

$$
\lim _{\varepsilon \rightarrow 0} \int_{C_{\varepsilon}} f(\zeta) \wedge R(\zeta, z) \wedge \varphi(z)=0
$$


where

$$
R(\zeta, z)=(1-G(\langle Q, \zeta-z\rangle))\langle s, \zeta-z\rangle^{-(n-p)} \operatorname{det}[\overbrace{h_{l j}}^{1 \leq l \leq p}, s_{j}, \overbrace{\bar{\partial} s_{j}}^{n-p-1}] \wedge \beta(\zeta) .
$$

To prove (1) observe that

$$
\left|\tilde{A}_{k}(\zeta, a)\right|=O\left(|\zeta-z|\left(\frac{1}{|\zeta-z|^{2}}\right)^{n-p-1}\right)=O\left(|\zeta-z|^{-(2 n-2 p)+3}\right)
$$

uniformly in $\zeta \in \bar{M}$ and $z$ in compact subsets of $M$; this follows from the assumptions made about $s$ and from the fact that $k \geq 1$. Hence

$$
\left|\tilde{A}_{k}(\zeta, z)\right|=O\left(\varepsilon^{-(2 n-2 p)+3}\right)
$$

uniformly in $(\zeta, z) \in C_{\varepsilon} \cap\left(M \times \operatorname{supp}(\varphi)\right.$. But $C_{\varepsilon}=\cup_{\zeta \in M}\{(\zeta, z): z \in M$, $|\zeta-z|=\varepsilon\}$ and by Fubini's Theorem

$$
\operatorname{Vol}\left(C_{\varepsilon}\right)=O\left(\varepsilon^{2 n-2 p-1}\right)
$$

(here Vol means $(4 n-4 p-1)$-dimensional Hausdorff measure). Let us also keep in mind that $\varepsilon$ goes to 0 through $\varepsilon>0$ so that $C_{\varepsilon}$ is a smooth $(4 n-4 p-1)$-dimensional manifold; that can be done in view of Sard's Theorem.

From (3) and (4) we obtain

$$
\int_{C_{\varepsilon}} f \wedge \tilde{A}_{k} \wedge \varphi=O\left(\varepsilon^{2}\right) \quad(k \geq 1)
$$

which implies (1).

The proof of (2) is similar; indeed, by $G(0)=1$ we obtain

$$
|1-G(\langle Q, \zeta-z\rangle)|=O(|\langle Q, \zeta-z\rangle|)=O(|\zeta-z|)
$$

and therefore

$$
|R|=O\left(|\zeta-z|^{(-2 n-2 p)+2}\right)
$$

Thus, as before, we obtain

$$
\int_{C_{\varepsilon}} f \wedge R \wedge \varphi=O(\varepsilon)
$$

which gives (2).

This completes the reduction to the case $Q \equiv 0$. This means that, in order to prove $(I)$ it remains to prove

$$
\lim _{\varepsilon \rightarrow 0} \int_{C_{\varepsilon}} f \wedge L^{s} \wedge \varphi=\int_{z \in M} f(z) \wedge \varphi(z)
$$


where $L_{s}$ is $K$ with $Q \equiv 0$, that is,

$$
L^{s}=c\langle s, \zeta-z\rangle^{-(n-p)} \operatorname{det}[h_{1 j}, \ldots, h_{p j}, s_{j}, \overbrace{\bar{\partial} s_{j}}^{n-p-1}] \wedge \beta(\zeta) .
$$

Now we will show that to prove $\left(I I_{s}\right)$ it suffices to prove $\left(I I_{b}\right)$ (recall $b_{j}=$ $\bar{\zeta}_{j}-\bar{z}_{j}$ ). For this we need the following lemma.

Lemma 3. For $(\zeta, z, \zeta) \in \bar{M} \times \bar{M} \times \mathbb{C}^{n}$ with $\langle\zeta, \zeta-z\rangle \neq 0$ define $\mu$ as follows:

$$
\mu(\zeta, z, \zeta)=\langle\zeta, \zeta-z\rangle^{-(n-p)} \operatorname{det}[h_{1 j}, \ldots, h_{p j}, \zeta_{j}, \overbrace{d \zeta}^{n-p-1}] \wedge \beta(\zeta) .
$$

Then $d[\mu \wedge \beta(z)]=d_{\zeta, z, \zeta}[\mu \wedge \beta(z)]=0$.

The proof of lemma 3 is similar to that of Lemma 2 (in fact it is simpler as it corresponds to the case $Q \equiv 0$; see also [8, Lemma 1])

Reduction of $\left(I I_{s}\right)$ To $\left(I I_{b}\right)$. First we may assume that $\langle s, \zeta-z\rangle>0$ (for $\zeta \neq z$ ) since

$$
L^{s}=L^{s^{*}}
$$

where $s^{*}=(\overline{\langle s, \zeta-z\rangle} /|\langle s, \zeta-z\rangle|) s$ and $\left\langle s^{*}, \zeta-z\right\rangle=|\langle s, \zeta-z\rangle|$. Define $\eta: \bar{M} \times \bar{M} \times[0,1] \rightarrow \bar{M} \times \bar{M} \times \mathbb{C}^{n}$ by $\eta(\zeta, z, \lambda)=\left(\zeta, z, s_{\lambda}\right)$ where $s_{\lambda}=\lambda s+(1-\lambda) b$. Let $N=\eta^{*}[\mu]$ be the pullback of $\mu$ via $\eta$. It follows from Lemma 3 that $\partial^{\prime} N=:\left(d_{\zeta}+\bar{\partial}_{z}+d_{\lambda}\right) N=0$. Now consider the integral

$$
J_{\varepsilon}=\int_{\partial\left(C_{\varepsilon} \times[0,1]\right)} f \wedge N \wedge \varphi
$$

By Stokes's Theorem

$$
J_{\varepsilon}= \pm \int_{C_{\varepsilon} \times[0,1]} N \wedge d(f \wedge \varphi)
$$

(here we use the equation $\partial^{\prime} N=0$ ). Also

$$
c J_{\varepsilon}=\int_{C_{\varepsilon}} N \wedge L^{s} \wedge \varphi-\int_{C_{\varepsilon}} f \wedge L^{b} \wedge \varphi
$$

provided that $\varepsilon>0$ is small enough; this follows the fact that $\varphi$ has compact support in $M$.

But by degree reasons the integral in (1) can be written as

$$
J_{\varepsilon}= \pm \int_{C_{\varepsilon} \times[0,1]} \tilde{N} \wedge d(f \wedge \varphi)
$$


where $\widetilde{N}$ is that part of $N$ which contains the differential $d \lambda$, that is, $\widetilde{N}=($ constant $)\left\langle s_{\lambda}, \zeta-z\right\rangle^{-(n-p)} \operatorname{det}[\overbrace{h_{l j}}^{1 \leq l \leq p},\left(s_{\lambda}\right)_{j},\left(b_{j}-s_{j}\right) d \lambda, \overbrace{\bar{\partial}\left(s_{\lambda}\right)_{j}}^{n-p-2}] \wedge \beta(\zeta)$. Hence, since $\left\langle s_{\lambda}, \zeta-z\right\rangle=\lambda\langle s, \zeta-z\rangle+(1-\lambda)|\zeta-z|^{2}$ and $\langle s, \zeta-z\rangle>0$,

$$
\tilde{N}=O\left(|\zeta-z|^{2} \cdot\left(|\zeta-z|^{-2}\right)^{n-p}\right)=O\left(|\zeta-z|^{-(2 n-2 p)+2}\right)
$$

and therefore by (2) we obtain that $J(\varepsilon)=O(\varepsilon)$. Thus

$$
\lim _{\varepsilon \rightarrow 0} J(\varepsilon)=0, \quad \text { that is, } \quad \lim _{\varepsilon \rightarrow 0} \int_{C_{\varepsilon}} N \wedge L^{s} \wedge \varphi=\lim _{\varepsilon \rightarrow 0} \int_{C_{\varepsilon}} N \wedge L^{b} \wedge \varphi .
$$

Therefore the reduction of $\left(I I_{s}\right)$ to $\left(I I_{b}\right)$ has been established.

COMPLETION OF THE PROOF OF THEOREM 1 . Theorem 1 has already been reduced to $\left(I I_{s}\right)$ which, in turn, has been reduced to $\left(I I_{b}\right)$. But $\left(I I_{b}\right)$ is exactly what is proved in the proof of [8, Theorem 1]. This completes the proof of Theorem 1 .

\section{References}

[1] I. Aizenberg and A. Yuzhakov, Integral representations and residues in multidimensional complex analysis., Trans. Math. Monographs 58, Amer. Math. Soc., Providence, R. I., 1983.

[2] M. Andersson and B. Berndtsson, Henkin-Ramirez formulas with weight factors, Ann. Inst. Fourier Grenoble 32 (3) (1982), 91-110.

[3] B. Berndtsson, A formula for interpolation and division in $\mathbb{C}^{n}$, Math. Ann. 263 (1983), 399-418.

[4] B. Berndtsson, Integral formulas on projective space and the Radon transform of Gindikin-Henkin-Polyakov, Pub. Mat. 32 (1988), 7-41.

[5] P. Charpentier, Solutions minimales de l'equation $\bar{\partial} u=f$ dans la boule et dans le polydisque, Ann. Inst. Fourier 30 (4) (1980), 121-153.

[6] S. V. Dautov and G. M. Henkin, Zeros of holomorphic functions of finite order and weighted estimates for solutions of the $\bar{\partial}$-equation, Math. USSR-Sb. 107 (1979), 163174.

[7] T. Hatziafratis, Integral representation formulas on analytic vanities, Pacific J. Math. 123 (1986), 71-91.

[8] T. Hatziafratis, An explicit Koppleman type integral formula on analytic varieties, Michigan Math. J. 3 (1986) 335-341.

[9] G. M. Henkin and J. Leiterer, Theory of functions on complex manifolds, (Birkhäuser, 1984).

[10] G. M. Henkin and J. Leiterer, Andreotti-Grauert theory by integral formulas, (Birkhäuser, 1988).

[11] L. Hörmander, An introduction to complex analysis in several variables, (North-Holland, k1973). 
[12] N. Ovrelid, Integral representation formulas and $L^{p}$-estimates for the $\bar{\partial}$-equation, Math. Scand. 29 (1971), 137-160.

[13] R. M. Range, Holomorphic functions and integral representations in several complex variables, (Springer Verlag, 1986).

[14] H. Skoda, (Valerus au bord pour les solutions se l'opérateur d"et caractérisation des zéros des fonctions de la classe de Nevanlinna), Bull. Soc. Math. France 104 (1976), 225-299.

\section{University of Athens}

\section{Athens}

\section{Greece}

